\title{
Being Rational About Health: The Pandemic's Long-Term Silver Lining?
}

\author{
Aubrey D.N.J. de Grey
}

Generals are always prepared to fight the last war. -Winston Churchill

$\mathbf{I}$ BRIEFLY TOYED WITH THE IDEA of writing an editorial that does not focus on the current global pandemic, but, well...

Many years ago I discussed in this space ${ }^{1}$ a scenario that, while fanciful, bears some resemblance to our prevailing predicament: the evolution of HIV into a form transmissible by air, resulting in essentially everyone contracting it because of its long latency. I explored how society might react, and contrasted it with the way we react to another medical condition that I need not name, which everyone already has but which society somehow does not take quite so seriously.

The bulk of our attention presently focuses, quite rightly, on the near-term priority of overcoming Covid-19 and minimizing the loss of life that it is causing. However, it is never too soon to allocate some of our thoughts and actions to forward planning: to ensuring that our post-crisis actions do not merely revert to the status quo ante, but are refined in such a way as to minimize the risk of similar future crises. This is, as a rule, too much to ask when we refer to society at large, but it may not be so when considering actions of governments-especially those actions that occur behind the scenes. The most obvious example of success in this regard is the reaction to 9/11: the measures that have been most conspicuous to the public in minimizing subsequent terrorist activity and those that have been the most effective in doing so are very probably not strongly correlated.

Prevention has not been the strong suit of medical practice over the years, despite the public's general acceptance in principle of the dictum that it is better than cure. In one sense this is not the medical community's fault: in essence, it arises from society's entirely correct impression that medicine is not all that far removed from magic, i.e. that medics only very approximately know what they're doing and can easily do more harm than good, especially with experimental new treatments. If the amount of good that can be done is seen as slight, simply because one is not yet sick, the risk/benefit ratio is not seen as attractive. And the logic that invalidates that thinking is quite hard to communicate, relying as it does on an assessment of the "discount rate" of the importance of one's health at greater or lesser distances into the future.

Hence the need to consider measures that do not require public action or decision-making, only assent (to the extent that they are even public_-but I won't go there today). One category of such measures, including the addition of fluoride to drinking water or folate to flour, does not merit much discussion in this forum, though it surely has great relevance to the broader issue of public health. My focus here, instead, is on what changes might be appropriate-and, thus, might be worth advocating for before decision-makers start to decide that they already know what to do-in the realm of policies that are relatively inexpensive and inconspicuous but yet highly impactful. Among these, I would like to highlight three, though without in any way implying that no others are comparably important.

Firstly and most obviously, it is vital to develop and maintain infrastructure to maximize readiness to respond to the emergence of a new infection, with particular emphasis on those that are transmissible before they become symptomatic. As I write, it remains the expert consensus that no vaccine against Covid-19 will be widely available until late this year at the earliest. To take just one example: can the pipeline for developing, testing, and manufacturing monoclonal neutralizing antibodies be greatly streamlined with a suitable amount of public investment? I bet it can. More generally, and I do not in any sense claim originality for this sentiment, it is an utter scandal that vaccine and antibiotic development has historically received such paltry public funding. If that neglect can be brought to an end, or even substantially alleviated, in the aftermath of the pandemic, that will constitute a very meaningful silver lining.

Secondly, and most directly relevant to the current public conversation: while any infection is preferentially dangerous for those with weak immune systems, different infections require subtly different strengths - and it seems clear, from the statistics that most of us are monitoring day by day, that the aspects of the immune response that are of greatest relevance to Covid-19's potency overlap greatly with those that most severely decline with age. Accordingly, there is an intense need to bring to policy-makers' attention the

SENS Research Foundation, Mountain View, California. 
immense potential for recent research advances to bear fruit as genuine rejuvenation of the immune systems of the elderly. If vaccines can be made to work even half as well in the older population as they do for young adults, we will be in a different world. Now is the time to communicate the message that that world is truly foreseeable.

Finally, and fittingly for an editorial in $R R$, there is a more general message. The immune system is just one of the many functions of our bodies that become impaired with age, but it is a particularly pervasive one: immunosenescence results not only in diminished defense against infections, but also in weakened ability to overcome incipient cancers and in elevated autoimmune disease. As such, if the current pandemic can educate decision-makers as to the need to invest more wisely and farsightedly in medical research and infrastructure, it has the potential to extend to a sea change in how such people think about public health in general. For too long, the pandemic of aging has been viewed as politically toxic, with greater concern being assigned to the economics of paying pensions than to the humanitarian imperative to maximize the health of all age groups. From this massive crisis, like other crises of the past, there may arise an opportunity. Those with influence in the corridors of power have a duty not to waste it.

\section{References}

1. de Grey ADNJ. Aging and airborne HIV: a reassuring analogy. Rejuvenation Res 2007;10:1-3.

Address correspondence to: Aubrey D.N.J. de Grey SENS Research Foundation Mountain View, CA 94041

E-mail: aubrey@sens.org 